\title{
Indication for Different Mechanisms of Kidney Uptake of Radiolabeled Peptides
}

\author{
Martin Gotthardt*1, Julliëtte van Eerd-Vismale*1, Wim J.G. Oyen ${ }^{1}$, Marion de Jong ${ }^{2}$, Hanwen Zhang ${ }^{3}$, \\ Edgar Rolleman ${ }^{2}$, Helmut R. Maecke ${ }^{3}$, Martin Béhé ${ }^{4}$, and Otto Boerman ${ }^{1}$ \\ ${ }^{I}$ Department of Nuclear Medicine, Radboud University Nijmegen Medical Center, Nijmegen, The Netherlands; ${ }^{2}$ Department of Nuclear \\ Medicine, Erasmus University Rotterdam Medical Center, Rotterdam, The Netherlands; ${ }^{3}$ Division of Radiological Chemistry, \\ Department of Radiology, Institute of Nuclear Medicine, University Hospital, Basel, Switzerland; and ${ }^{4}$ Department of Nuclear \\ Medicine, University Hospital Giessen and Marburg, Campus Marburg, Marburg, Germany
}

Nephrotoxicity due to renal reabsorption of radiolabeled peptides limits the tumor dose in peptide receptor radiotherapy (PRRT). Therefore, we evaluated the ability of several agents to inhibit the renal accumulation of different radiopeptides. Methods: Male Wistar rats (4 per group) were injected intravenously with $1 \mathrm{MBq}$ of ${ }^{111} \mathrm{In}$-labeled octreotide (OCT), minigastrin (MG), bombesin (BOM), or exendin (EX), together with a potential inhibitor of renal uptake (lysine [Lys], poly-glutamic acid [PGA], and Gelofusine [GF], a gelatin-based plasma expander) or phosphatebuffered saline as a control. Organ uptake at $20 \mathrm{~h}$ after injection was determined as the percentage of injected activity per gram $(\% \mathrm{IA} / \mathrm{g})$. Lys, PGA, and GF were also combined to determine whether an additive effect could be obtained. The localization of the peptides in the kidneys was investigated by autoradiography using a phosphor imager. Results: OCT accumulation in the kidney was inhibited by Lys and GF (40.7\%-45.1\%), whereas PGA was ineffective. On the other hand, renal uptake of BOM, $M G$, and EX was inhibited by PGA and GF (15.4\%-85.4\%), whereas Lys was ineffective. The combination of GF and Lys showed additive effects in inhibiting OCT uptake, whereas PGA and GF had additive effects for the inhibition of EX uptake. The amount of kidney uptake correlated with the number of charged amino acids. All radiopeptides were localized in the renal cortex, as indicated by autoradiography. Conclusion: Inhibition of renal accumulation of the radiopeptides tested could be achieved by either Lys or PGA but not by both at the same time, suggesting 2 different uptake mechanisms. The differences in renal accumulation of radiopeptides may be related to the number of charges of a molecule. GF is the only compound that inhibited renal accumulation of all radiopeptides tested. Additional experiments are needed to further elucidate these findings and to optimize inhibition of renal accumulation of radiopeptides to reduce the kidney dose in PRRT.

Key Words: radiopeptide; kidney; reabsorption; peptide receptor radiotherapy

J Nucl Med 2007; 48:596-601

DOI: 10.2967/jnumed.106.036020

Received Aug. 24, 2006; revision accepted Dec. 15, 2006.

For correspondence or reprints contact: Martin Gotthardt, MD, PhD, Department of Nuclear Medicine, Radboud University Nijmegen Medical Center, Postbus 9101, 6500 HB Nijmegen, The Netherlands.

E-mail: m.gotthardt@nucmed.umcn.nl

${ }^{*}$ Contributed equally to this work.

COPYRIGHT @ 2007 by the Society of Nuclear Medicine, Inc.
$\mathbf{I}$ n the tubules of the kidney, small proteins and polypeptides are reabsorbed after glomerular filtration. An important mechanism of reabsorption is uptake via megalin, a 660-kDa transmembrane scavenger-receptor, which has been shown to be responsible for kidney accumulation of radiolabeled octreotide (OCT) $(1,2)$. Renal reabsorption of radiolabeled peptides or antibody fragments is a clinical problem because, on one hand, the kidney is the dose-limiting organ in peptide receptor radiotherapy (PRRT) and, on the other hand, high kidney uptake may obscure pathologic findings in adjacent structures in scintigraphic imaging. The problem of high kidney uptake of radiolabeled compounds may be overcome by coadministration of cationic amino acids that reduce the kidney uptake of both radiolabeled peptides and antibody fragments $(1,3)$. In PRRT, lysine (Lys) and arginine (Arg) are usually applied intravenously for reduction of kidney uptake $(4,5)$. Oral administration has been shown in animals to also reduce renal uptake of antibody fragments as well as radiopeptides (3,6). As an alternative to oral or intravenous application of Lys or Arg, gelatinbased plasma expanders (Gelofusine [GF], B Braun Medical BV; Hemaccel, Sanofi-Aventis Pharma BV) may be administered intravenously to patients $(7,8)$.

It is assumed that renal uptake of most peptides or small proteins can be inhibited by administration of cationic amino acids. However, renal accumulation of the radiopeptide ${ }^{111}$ In-D-Glu ${ }_{1}$-minigastrin (MG) cannot be inhibited by the cationic amino acid Lys or Arg (9). Recently, Béhé et al. showed that poly-glutamic acid (PGA) effectively reduced MG uptake in the kidneys. Effective inhibition was demonstrated for a chain length of 5 glutamic acid residues or more, whereas a chain length of $<5$ residues had no effect on renal uptake (9).

Our aim was to further investigate whether renal accumulation of radiopeptides other than OCT or MG could also be inhibited by GF or PGA, respectively. Therefore, we assessed the influence of different compounds known to inhibit kidney uptake of radiopeptides (Lys, PGA, GF) on renal accumulation of analogs of somatostatin (OCT), gastrin (MG), glucagonlike peptide-1 (exendin [EX]), and bombesin (BOM). 


\section{MATERIALS AND METHODS}

\section{Radiolabeling}

DTPA-D-Phe ${ }^{1}$-octreotide (DTPA is diethylenetriaminepentaacetic acid) (Octreoscan) was obtained from Tyco Health Care and labeled as described previously (7). Briefly, $\sim 40 \mathrm{MBq}^{111} \mathrm{InCl}_{3}$ were transferred to the reaction vial containing $10 \mu \mathrm{g}$ OCT. After incubation at room temperature for $30 \mathrm{~min}$, quality control was performed by $\mathrm{C}_{18}$ reverse-phase high-pressure liquid chromatography (RP-HPLC), using $0.1 \%$ trifluoroacetic acid, $\mathrm{pH} 7.4$, as mobile phase with a linear gradient rising to $70 \%$ acetonitrile over $30 \mathrm{~min}$ (flow rate, $1 \mathrm{~mL} / \mathrm{min}$; column, $4.6 \mathrm{~mm} \times 25 \mathrm{~cm}$; Hewlett Packard 1100 series automated HPLC system). Labeling of the DTPA-Glu ${ }^{-}$ minigastrin (MG; Sigma-Genosys) and DTPA-Lys $40^{-}$-Exendin 4 (EX; Peptide Specialty Laboratories $\mathrm{GmbH}$ ) was done essentially as described (10). In short, $\sim 40 \mathrm{MBq}^{111} \mathrm{InCl}_{3}$ in $100 \mu \mathrm{L}$ of $0.1 \mathrm{M}$ (MG) or $0.05 \mathrm{M}$ (EX) $\mathrm{HCl}$ were added to $100 \mu \mathrm{L}$ of the peptide dissolved at a concentration of $50 \mu \mathrm{M}$ in $0.25 \mathrm{M}$ ammonium acetate buffer, $\mathrm{pH} 5.0$, and incubated for $30 \mathrm{~min}$ at room temperature.

DOTA-Sar ${ }_{5}-\left[\mathrm{D}-\mathrm{Tyr}^{5,6}, \mathrm{BAla}^{11}, \mathrm{Thi}^{13}{ }^{13} \mathrm{Nle}^{14}\right] \operatorname{bombesin}(6-14)$ (BOM) was provided by Prof. Helmut Maecke. For labeling, $40 \mathrm{MBq}{ }^{111} \mathrm{InCl}_{3}$ were added to the reaction vial containing $100 \mu \mathrm{g}$ BOM dissolved in $0.25 \mathrm{M}$ ammonium acetate, $\mathrm{pH}$ 5.0. After incubation at $80^{\circ} \mathrm{C}$ for 60 min, quality control was performed using $\mathrm{C}_{18}$ RP-HPLC as described.

\section{Inhibitors of Tubular Reabsorption}

All chemicals were obtained from Sigma-Aldrich unless stated otherwise. Lys was used at a concentration of $160 \mathrm{mg} / \mathrm{mL}$ dissolved in phosphate-buffered saline (PBS). GF is a commercially available succinylated gelatin solution (40 g/L) (Braun). PGA (3-15 kDa) was used at a concentration of $80 \mathrm{mg} / \mathrm{mL}$ dissolved in PBS (9).

\section{Animal Studies}

Male Wistar rats (age, 4-8 wk; weight, $\sim 250 \mathrm{~g}$; Harlan Netherlands) were used in the experiments; 4 animals were used per group. All experiments had been approved by the local animal welfare committee and were performed according to the Dutch laws on laboratory animal welfare.

For all experiments, $1 \mathrm{MBq}$ of the respective ${ }^{111} \mathrm{In}$-labeled compound were injected intravenously into the tail vein per animal $(0.2 \mathrm{~mL})$. Immediately before injection of the radiopeptide, $0.5 \mathrm{~mL}$ of the Lys/Arg/PGA $(80 \mathrm{mg} / \mathrm{mL})$ solution or GF $(40 \mathrm{mg} / \mathrm{mL})$ were injected. In addition to injection of single substances, a combination of PGA and GF or Lys and GF in the same doses as stated here was used together with MG, EX, or OCT, respectively. Animals were euthanized $24 \mathrm{~h}$ after injection and dissected. In addition to a blood sample, heart, liver, lung, kidney, spleen, pancreas, and stomach were dissected, blotted dry, and weighed. Activity in the organs was measured using a $\gamma$-counter (Wallac), and the uptake was determined as the percentage of injected activity (\%IA) per gram of organ weight. In each experiment, a control group was preinjected with $0.5 \mathrm{~mL}$ of PBS before injection of the respective radiopeptide.

\section{Autoradiography}

To determine the localization of the respective radiopeptides after uptake into the kidney, autoradiography of kidney slices was performed after injection of $250 \mathrm{MBq}$ of the respective radiolabeled peptide (higher activity than in biodistribution studies to allow for shorter exposure times). Animals were euthanized and dissected $24 \mathrm{~h}$ after injection. The slices were cryofixed in TissueTek (Sacura Finetek) and snap-frozen in isopentane. Slices of $5 \mu \mathrm{m}$ were cut with a cryostatic microtome. The sections were air dried and exposed to a storage phosphor imager screen overnight. The screen was scanned in a phosphor imaging system (Molecular Imager GS363; BioRad Laboratories) at a pixel size of $100 \times 100$ $\mu \mathrm{m}$. Images were processed with Quantity One software (version 4.5.2; BioRad Laboratories).

\section{Statistical Analysis}

GraphPad Prism was used for statistical evaluation. Values are presented as the mean \pm SD. All data were analyzed by 2-way ANOVA, including Bonferroni testing if $>2$ groups were compared. Kidney uptake values after administration of inhibitors of kidney uptake were compared with the respective controls by a $t$ test (Mann-Whitney). The significance level was set to $P \leq 0.05$.

\section{RESULTS}

\section{Radiolabeling}

All peptides were labeled at a specific activity of 4-8 $\mathrm{GBq} / \mu \mathrm{mol}$. Radiochemical purity as determined by HPLC was $>95 \%$. Unbound ${ }^{111}$ In eluted from the column after 2-3 min, whereas retention time of the labeled peptides was 15-21 min, dependent on the respective peptide.

\section{Kidney Uptake Inhibition}

In all experiments, the uptake in tissues other than the kidney did not differ significantly between the control group and the groups that had received the potential inhibitors of the kidney accumulation of the respective radiopeptides (ANOVA). The influence of GF, PGA, Lys, and Arg on uptake of the different radiopeptides is summarized in Table 1. In the following, the results are described in detail. The differences in kidney uptake are given in percentage of the $\%$ IA/g values. The differences in uptake in the control groups (PBS) are probably based on differences in hydration of the animals and other factors (such as body weight, amount of food intake, genetic variability, etc.), which are not always simple to standardize. Such differences may occur in any experimental study (as well as in clinical studies). However, the significant reduction in uptake of the radiopeptides caused by coadministration of inhibitory substances is reproducible, independently of the absolute uptake in $\% \mathrm{IA} / \mathrm{g}$.

\section{OCT}

In comparison with the control group, GF was shown to reduce the kidney uptake by $40.7 \%(P<0.01)$, whereas Lys caused a reduction of $35 \%(P<0.01)$. The combination of GF and Lys reduced kidney uptake by $49.7 \%(P<0.01)$ and was more effective in comparison with Lys alone $(P<0.01)$ and $\mathrm{GF}$ alone $(P=0.01)$. The efficacy of uptake inhibition of GF and Lys did not differ significantly $(P=0.13)$ (ANOVA for all groups, $P<0.01)$. PGA did not reduce kidney uptake significantly $(P=0.14)$ (Figs. 1A and $1 \mathrm{~B})$. The data for Lys and GF are consistent with the results reported in the literature $(1,3,7)$.

\section{MG}

Renal accumulation of $\mathrm{MG}$ was significantly reduced by $85.4 \%$ using PGA $(P<0.01)$ and by $45 \%$ using GF 
TABLE 1

Overview of Influence of Different Substances on Renal Uptake of Radiopeptides

\begin{tabular}{|c|c|c|c|c|c|}
\hline Radiopeptide & Lys & GF & PGA & GF + Lys & $\mathrm{GF}+\mathrm{PGA}$ \\
\hline \multicolumn{6}{|l|}{ OCT } \\
\hline $0.97 \pm 0.18$ & $0.63 \pm 0.07$ & $0.57 \pm 0.05$ & $1.11 \pm 0.1$ & $0.49 \pm 0.05$ & Not done \\
\hline $1.33 \pm 0.28$ & $P<0.01$ & $P<0.01$ & $P=0.14$ & $\begin{array}{l}P<0.01 \text { (Lys) } \\
P=0.01 \text { (GF) }\end{array}$ & \\
\hline \multicolumn{6}{|c|}{ ( } \\
\hline $\begin{array}{l}12.16 \pm 1.78 \\
9.8 \pm 0.77\end{array}$ & $\begin{array}{c}12.11 \pm 1.04 \\
P=0.57\end{array}$ & $\begin{array}{c}6.69 \pm 0.78 \\
P<0.01 \\
6.89 \pm 0.18\end{array}$ & $\begin{array}{c}1.77 \pm 0.25 \\
P<0.01 \\
1.89 \pm 0.14\end{array}$ & Not done & $\begin{array}{c}1.95 \pm 0.18 \\
P<0.05(G F) \\
P=0.51(P G A)\end{array}$ \\
\hline \multicolumn{6}{|l|}{ BOM } \\
\hline $0.35 \pm 0.06$ & $\begin{array}{c}0.34 \pm 0.06 \\
P=0.51\end{array}$ & $\begin{array}{l}0.3 \pm 0.02 \\
P=0.005\end{array}$ & $\begin{array}{c}0.27 \pm 0.03 \\
P=0.038\end{array}$ & Not done & Not done \\
\hline \multicolumn{6}{|l|}{ EX } \\
\hline $20.52 \pm 1.86$ & $\begin{array}{c}21.86 \pm 2.57 \\
P=0.8\end{array}$ & $\begin{array}{c}16.68 \pm 1.01 \\
P<0.01\end{array}$ & $\begin{array}{c}14.48 \pm 1.43 \\
P<0.01\end{array}$ & Not done & $\begin{array}{c}12.08 \pm 1.01 \\
P<0.01 \text { (PGA, GF) }\end{array}$ \\
\hline
\end{tabular}

Kidney uptake values are given as mean $\pm \mathrm{SD}$ in \% IA/g. If results have not been obtained in 1 set of experiments, alternative results belonging together are given in italic. In the last 2 columns on the right, $P$ values indicate significance in comparison with respective single substances.

$(P<0.01)$, whereas Lys did not show a significant effect $(P=0.57)$ (Fig. 2A). In an additional experiment, the combination of identical concentrations of GF and PGA did not result in a further decrease of kidney accumulation of MG in comparison with PGA alone (PGA, $-80.8 \%[P<$ $0.01]$; GF, $-29.8 \%[P<0.01]$; $\mathrm{PGA}+\mathrm{GF}$ in comparison with $\mathrm{GF},-50.3 \%[P<0.05]$; $\mathrm{PGA}+\mathrm{GF}$ in comparison with PGA, $+0.7 \%[P=0.51]$ ) (Fig. $2 \mathrm{~B}$ ).

\section{BOM}

The kidney accumulation of BOM was very low in comparison with the other radiopeptides, with $<0.5 \% \mathrm{IA} / \mathrm{g}$. PGA as well as GF significantly reduced kidney accumulation (by $23.8 \%[P=0.038]$ and by $15.4 \%[P=0.005]$, respectively), whereas Lys did not demonstrate a significant influence $(P=0.51)$. The inhibitory effects of GF and PGA did not differ significantly $(P=0.11)$ (Fig. 3). Because of the low kidney uptake of BOM, a combination of PGA and GF was not tested for this peptide.

\section{EX}

Although Lys did not affect kidney uptake of EX (increase of $6.5 \%, P=0.8)$, GF and PGA lead to a decrease $(18.7 \%$ $[P<0.01]$ and $29.4 \%[P<0.01]$, respectively). The combination of PGA and GF decreased the uptake by 47.9\% ( $P<0.01)$ (Fig. 4). In comparison with GF and PGA alone, the combination of both agents at the same concentrations did result in a statistically significant additional reduction of uptake (in comparison with GF alone, $-27.6 \%[P<0.01]$, and in comparison with PGA alone, $-16.6 \%[P<0.01])$.

\section{Autoradiography}

Autoradiography of kidney sections showed localization of all radiopeptides in the cortex of the kidney (Figs. 5A5D). Distribution of activity shows the same pattern for all 4 radiopeptides.

\section{DISCUSSION}

It is well known that tubular reabsorption of peptides and antibody fragments can be inhibited by coadministration of cationic amino acids, such as Lys or Arg $(11,12)$. For OCT as a radiolabeled peptide, this effect was demonstrated in the early 1990s (13). It is assumed that uptake of OCT occurs in the proximal tubule cells in the kidneys. OCT accumulated in the proximal tubules and colocalized with megalin staining (2). In kidney-specific megalin knock-out mice, OCT uptake into the kidney is nearly absent. Therefore,
FIGURE 1. (A) Renal accumulation of OCT in \%IA/g $24 \mathrm{~h}$ after injection. Lys and GF significantly inhibit renal accumulation of OCT. Combination of both is more efficient in comparison with the single substances. (B) PGA does not show significant reduction of OCT uptake, whereas Lys (serving as control) does.

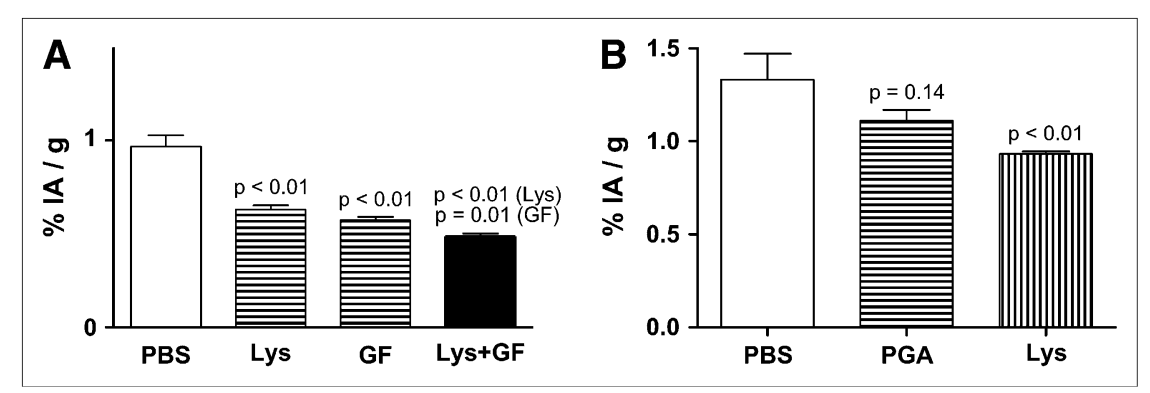




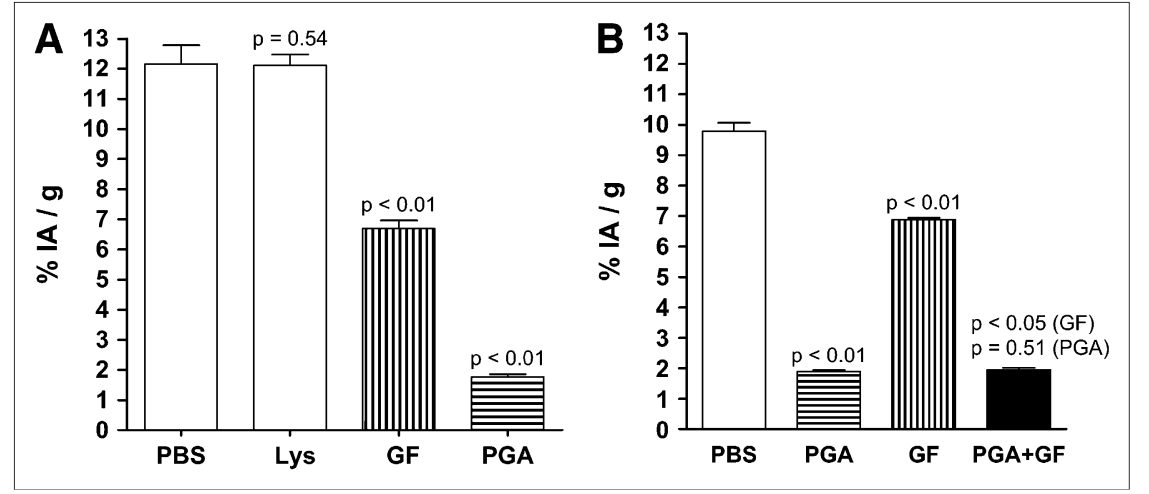

FIGURE 2. (A) Renal accumulation of $M G$ in \%IA/g $24 \mathrm{~h}$ after injection. GF and PGA significantly inhibit tubular accumulation of MG, whereas Lys does not. (B) Combination of GF and PGA does not exceed efficacy of PGA alone. megalin is thought to play a major role in renal accumulation of OCT $(1,2)$. The contribution of fluid-phase endocytosis to renal accumulation of OCT (14) needs to be further elucidated, although the data of Thelle et al. showing that Lys disturbs the endocytic system support the idea that it may play a role in addition to megalin (15). Several studies demonstrated that megalin binds proteins rich in positively charged amino acids and cationic compounds $(16,17)$. The hypothesis that Lys and Arg saturate those binding sites and, therefore, interfere with renal reabsorption of OCT is consistent with these findings $(18,19)$. However, recently, gelatin-based plasma expanders inducing proteinuria (comparable with that observed in megalindeficient mice) have also been found to reduce renal uptake of OCT $(7,8,20-22)$. Though cationic amino acids contained in GF may be responsible for this finding (23), it cannot be excluded that megalin is also able to bind other than cationic compounds via alternative binding mecha-

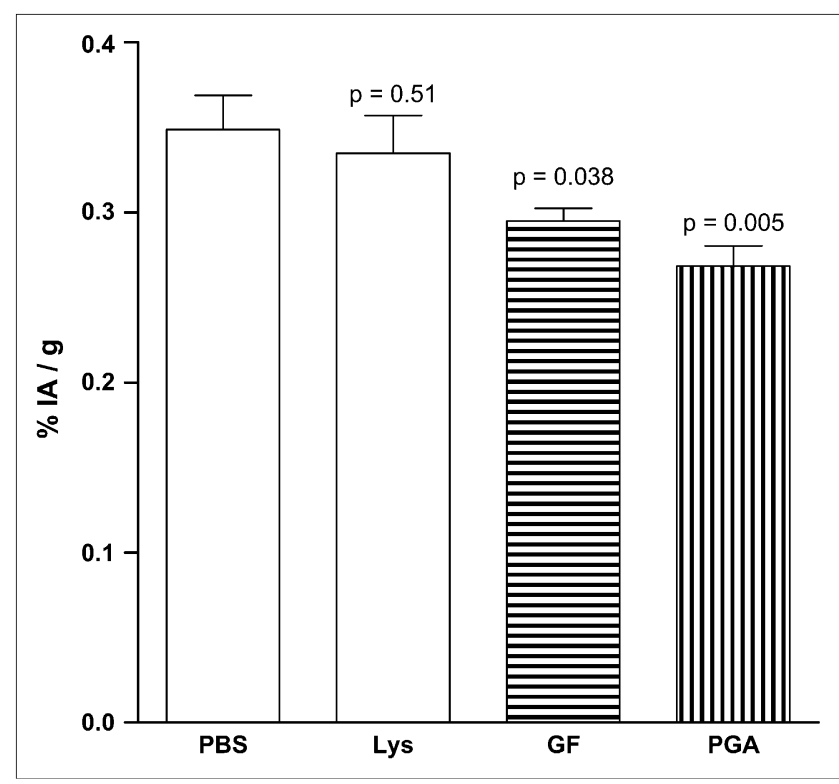

FIGURE 3. Renal accumulation of BOM in \%IA/g $24 \mathrm{~h}$ after injection. BOM shows pattern comparable to that of $M G$ as renal uptake can be reduced by PGA and GF, whereas Lys is not effective. nisms (7). GF is not the only noncationic compound demonstrating inhibitory effects on renal accumulation of radiopeptides. For MG, inhibition of renal accumulation by the anionic compound PGA of a chain length of $>4$ Glu residues has been demonstrated (9). However, in contrast to GF, it is unclear whether PGA may demonstrate significant side effects or even toxicity (in analogy to poly-Lys (3)), and its efficacy has not been proven in humans. GF may thus be a promising compound for inhibition of renal uptake of MG and other radiopeptides. The aim of our work was primarily to test whether renal accumulation of radiopeptides other than OCT could also be inhibited by GF and by the other known compounds that can reduce renal accumulation of radiopeptides. MG and EX could be considered for PRRT of neuroendocrine tumors as an alternative to OCT if somatostatin receptor expression is low or absent $(24,25)$. However, their high renal uptake would prevent their use for PRRT with ${ }^{177} \mathrm{Lu}$ or ${ }^{90} \mathrm{Y}$ because of the expected renal toxicity limiting

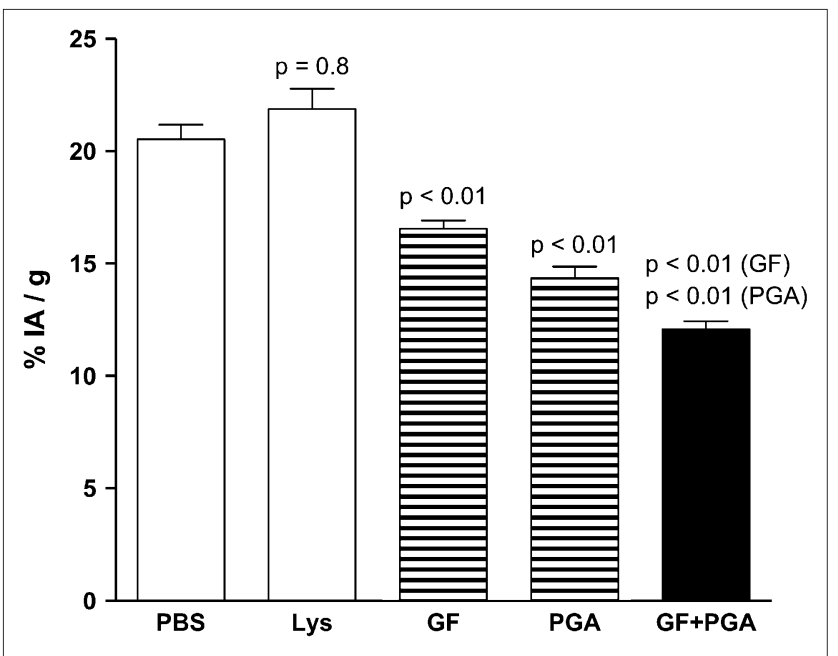

FIGURE 4. Renal accumulation of $E X$ in $\% \mathrm{IA} / \mathrm{g} 24 \mathrm{~h}$ after injection. EX shows same pattern of uptake inhibition as MG and BOM, indicating that uptake mechanism may be the same. Combination of PGA and GF shows additive effect (significant in comparison with single substances). 
A

FIGURE 5. Results of kidney autoradiography: (A) OCT, (B) MG, (C) EX, (D) BOM (from left to right). All compounds are colocalized in renal cortex; image quality reflects renal uptake of respective compounds.
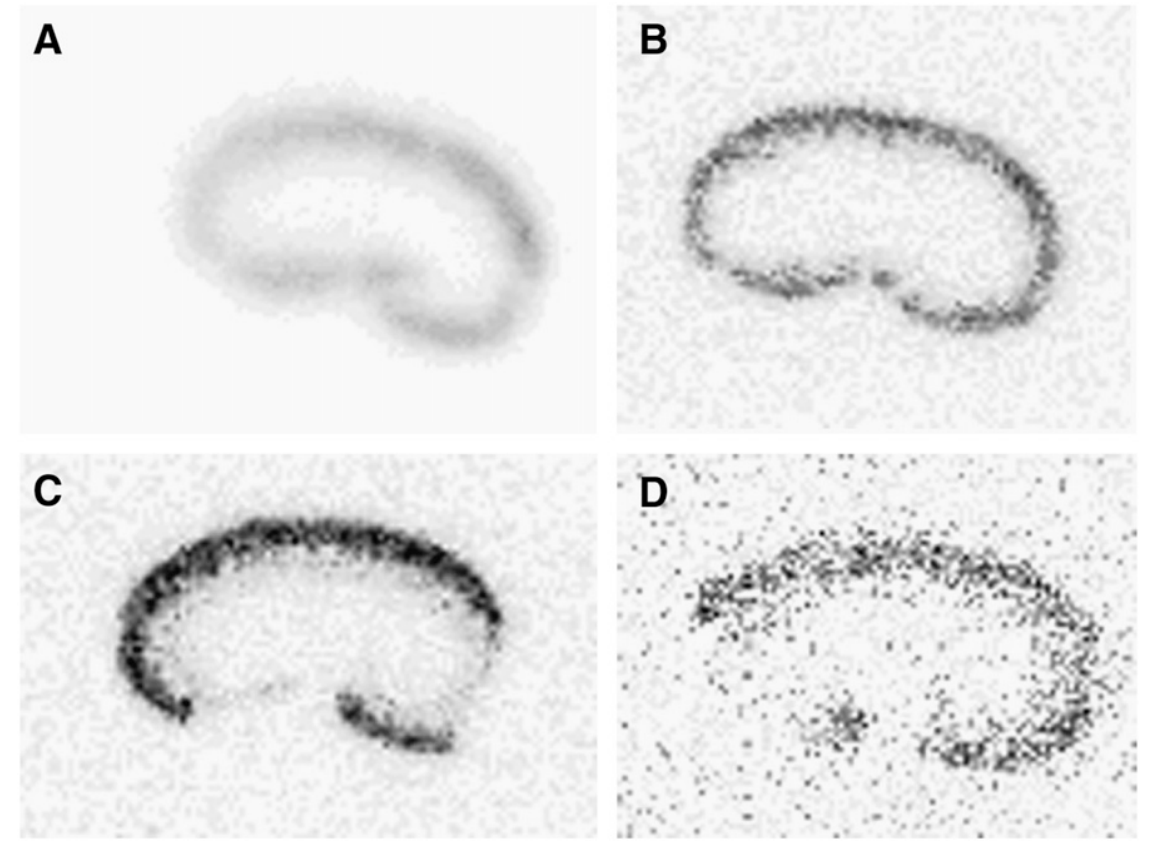

the applicable activities. Therefore, effective inhibition of renal accumulation of these compounds is warranted.

Our results indicate that inhibition of the uptake of OCT is different from that of the other radiopeptides tested. Renal uptake of OCT could be reduced by GF as well as Lys, whereas PGA did not exert an inhibitory action. These results are in line with reports from the literature $(7,9)$. It is interesting that PGA also reduced renal accumulation of EX and not only that of MG. However, MG uptake was more efficiently inhibited by PGA than uptake of EX. Furthermore, GF was able to reduce renal uptake of all peptides tested, whereas Lys reduced only OCT uptake. The latter findings are in accordance with the observation of Thelle et al. that Lys does not influence tubular reabsorption of rat urinary proteins 1 and 2 (15), thus indicating that not all proteins are taken up by the mechanism that can be inhibited by Lys.

The reduction of BOM uptake by $\mathrm{PGA}$ and GF was significant. However, BOM uptake is very low, indicating that the uptake mechanism is not very efficient or may not even be specific (thus, may be based on fluid-phase endocytosis alone). Lys, however, did not show any effect at all, although it has been demonstrated that Lys may disturb the entire endocytotic process (15). Therefore, our findings that PGA and GF slightly reduce kidney uptake of BOM also point toward involvement of different uptake mechanisms. However, the low baseline uptake together with the variability of the kidney uptake need to be kept in mind.

Therefore, our results suggest that different binding mechanisms for reabsorption of peptides or proteins in the kidney may exist. Autoradiography demonstrated that all tested radiopeptides accumulate in the renal cortex, which points toward a (proximal) tubular mechanism for all (which has been characterized for OCT (2)). Four different clusters of anionic amino-acid repeats that serve as binding sites of megalin for cationic molecules are described in the literature (19). Our results indicate that binding mechanisms for other molecules may also be present. These may interact with peptides with different properties (size, structure, charge, etc.) and not only with cationic peptides. If so, GF may at the same time block different binding mechanisms that are responsible for reabsorption of OCT as well the other radiopeptides, whereas PGA and Lys selectively block 1 binding mechanism only. The amount of kidney uptake of the peptides evaluated in our experiments showed a relationship with the number of charged groups of amino acids of the respective molecules. The order of kidney uptake was BOM $(\sim 0.35$ $\% \mathrm{IA} / \mathrm{g})<\mathrm{OCT}(\sim 1.5 \% \mathrm{IA} / \mathrm{g})<\mathrm{MG}(\sim 12 \% \mathrm{IA} / \mathrm{g})<\mathrm{EX}$ ( $\sim 20 \% \mathrm{IA} / \mathrm{g}$ ), whereas the number of charged amino acids (Glu, Lys, Asp, Arg) is 0 for BOM, $1(+)$ for OCT, 7 (-) for $\mathrm{MG}$, and 10 (4+, 6-) for EX. One may therefore hypothesize that renal reabsorption of peptides may be determined by the number of charged groups of amino acids. Furthermore, MG has the highest number of negative charges (6 Glu, 1 Asp) and no positive charges and can most effectively be inhibited by PGA. Other factors such as the chain length and the distribution of charges may also play a role. GF seems to be able to inhibit renal uptake of both cationic and anionic peptides. If this hypothesis is right, this would indicate that GF contains positive and negative charges and is able to inhibit renal accumulation of anionic and cationic peptides.

Our findings suggest that some inhibitors act by 2 different mechanisms to block renal reabsorption (GF), whereas others are specific for just 1 mechanism (Lys and PGA). A similar behavior of ligands of the megalin/cubilin system has been described. Some ligands, such as lysozyme, cytochrome $c$, or prolactin, are specific for megalin; others show specificity for cubilin only (e.g., transferrin, clara cell secretory protein), whereas others have affinity for both cubilin 
and megalin (e.g., albumin, hemoglobin, myoglobin) (19). Therefore, it is possible that renal reabsorption of radiopeptides also involves a second transport molecule apart from megalin. It remains unclear whether this may be cubilin and whether it may be responsible for binding of anionic peptides only. These questions need to be addressed in future experiments.

As a wide variety of peptides and proteins needs to be reabsorbed in the kidney to prevent loss of valuable amino acids, an effective physiologic mechanism is present to bind many different peptides or proteins that are filtered via the glomeruli (16). On the basis of these results, we postulate that such a reabsorption mechanism for peptides may be based on charged amino acids. Further experiments are warranted to support this hypothesis.

\section{CONCLUSION}

Inhibition of renal accumulation of radiopeptides can decrease nephrotoxicity in PRRT, and this can be achieved by administration of cationic compounds such as Lys or Arg. Our data show that inhibition of renal accumulation of radiopeptides may be more complex and that a mechanism that can be inhibited by anionic molecules also exists. It is not clear, however, whether megalin is also involved in renal uptake of anionic molecules. GF was able to inhibit renal uptake of all tested radiopeptides in our experiments and may, therefore, interact with both mechanisms. It is not totally clear which and how many components of GF are responsible for this effect (7). Therefore, further characterization of GF itself and of the mechanisms of renal accumulation of radiopeptides in general is needed to optimize inhibitors of renal accumulation of radiopeptides to achieve higher tumor doses in PRRT.

\section{REFERENCES}

1. Melis M, Krenning EP, Bernard BF, Barone R, Visser TJ, de Jong M. Localisation and mechanism of retention of radiolabelled somatostatin analogs. Eur J Nucl Med Mol Imaging. 2005;32:1136-1143.

2. de Jong M, Barone R, Krenning EP, et al. Megalin is essential for renal proximal tubule reabsorption of ${ }^{111}$ In-DTPA-octreotide. J Nucl Med. 2005;46:1696-1700.

3. Behr TM, Sharkey RM, Juweid ME, et al. Reduction of the renal uptake of radiolabeled monoclonal antibody fragments by cationic amino acids and their derivatives. Cancer Res. 1995;55:3825-3834.

4. Rolleman EJ, Valkema R, de Jong M, Kooij PP, Krenning EP. Safe and effective inhibition of renal uptake of radiolabeled octreotide by a combination of lysine and arginine. Eur J Nucl Med Mol Imaging. 2003;30:9-15.

5. Jamar F, Barone R, Mathieu I, et al. ${ }^{86}$ Y-DOTA(0)-D-Phe1-Tyr3octreotide (SMT 478): a phase 1 clinical study-pharmacokinetics, biodistribution, and renal protective effect of different regimens of amino acid co-infusion. Eur $J$ Nucl Med Mol Imaging. 2003;30:510-518.

6. Verwijnen SM, Krenning EP, Valkema R, Huijmans JG, de Jong M. Oral versus intravenous administration of lysine: equal effectiveness in reduction of renal uptake of [ ${ }^{111}$ In-DTPA] octreotide. J Nucl Med. 2005;46:2057-2060.

7. van Eerd JEM, Vegt E, Wetzels JFM, et al. Gelatin-based plasma expander effectively reduces renal uptake of ${ }^{111}$ In-octreotide in mice and rats. J Nucl Med. 2006;47:528-533.

8. Vegt E, Wetzels JF, Russel FG, et al. Renal uptake of radiolabeled octreotide in human subjects is efficiently inhibited by succinylated gelatin. J Nucl Med. 2006; 47:432-436.

9. Behe M, Kluge W, Becker W, Gotthardt M, Behr TM. Use of polyglutamic acids to reduce uptake of radiometal-labeled minigastrin in the kidneys. J Nucl Med. 2005;46:1012-1015.

10. Béhé M, Becker W, Gotthardt M, Angerstein C, Behr TM. Improved kinetic stability of DTPA-D-Glu as compared with conventional monofunctional DTPA in chelating indium and yttrium: preclinical and initial clinical evaluation of radiometal labelled minigastrin derivatives. Eur J Nucl Med Mol Imaging. 2003; 30:1140-1146.

11. Morgensen CE, Sølling K. Studies on renal tubular protein re-absorption: partial and near-complete inhibition by certain amino acids. Scand J Clin Lab Invest. 1977;37:477-486.

12. Pimm V, Gribben SJ. Prevention of renal tubule re-absortion of radiometal (indium-111) labelled Fab fragment of a monoclonal antibody in mice by systemic administration of lysine. Eur J Nucl Med. 1994;21:663-665.

13. Hammond PJ, Wade AF, Gwilliam ME, et al. Amino acid infusion blocks renal tubular uptake of indium-labelled somatostatin analogue. Br J Cancer. 1993;67: 1437-1439.

14. Barone R, van der Smissen P, Devuyst O, et al. Endocytosis of the somatostatin analogue, octreotide, by the proximal tubule-derived opossum kidney (OK) cell line. Kidney Int. 2005;67:969-976.

15. Thelle K, Christensen EI, Vorum H, Ørskov H, Birn H. Characterization of proteinuria and tubular protein uptake in a new model of oral L-lysine administration in rats. Kidney Int. 2006;69:1333-1340.

16. Christensen EI, Birn H. Megalin and cubilin: multifunctional endocytic receptors. Nat Rev Mol Cell Biol. 2002;3:256-266.

17. Moestrup SK, Cui S, Vorum H, et al. Evidence that epithelial glycoprotein 330/ megalin mediates uptake of polybasic drugs. J Clin Invest. 1995;96:1404-1413.

18. Trejtnar F, Laznicek M. Analysis of renal handling of radiopharmaceuticals. $Q J$ Nucl Med. 2002;46:181-194.

19. Nagai J, Takano M. Molecular aspects of renal handling of aminoglycosides and strategies for preventing the nephrotoxicity. Drug Metab Pharmacokinet. 2004; 19:159-170.

20. ten Dam MA, Branten AJ, Klasen IS, Wetzels JF. The gelatine-derived plasma substitute Gelofusien causes low-molecular-weight proteinuria by decreasing tubular protein reabsorption. J Crit Care. 2001;16:115-120.

21. Veldman BA, Schepkens HL, Vervoort G, Klasen I, Wetzels JF. Low concentrations of intravenous polygelines promote low-molecular weight proteinuria. Eur J Clin Invest. 2003;33:962-968.

22. Leheste JR, Rolinski B, Vorum H, et al. Megalin knockout mice as an animal model of low molecular weight proteinuria. Am J Pathol. 1999;155:1361-1367.

23. Kadler KE, Holmes DF, Trotter JA, Chapman JA. Collagen fibril formation. Biochem J. 1996;316:1-11.

24. Gotthardt M, Boermann OC, Behr TM, Béhé MP, van Oyen WJG. Development and clinical application of peptide-based radiopharmaceuticals. Curr Pharm Des. 2004;10:2951-2963.

25. Gotthardt M, Béhé M, Grass J, et al. Added value of gastrin receptor scintigraphy to somatostatin receptor scintigraphy in neuroendocrine tumors. Endocr Relat Cancer. 2006;13:1203-1211. 